\title{
Une histoire de machines à recommander des biens de consommation : de l'Internet documentaire à l'Internet des données
}

A History of Consumer Recommendation Machines: From the Internet of Documents to the Internet of Data

Jean-Sébastien Vayre

\section{OpenEdition Journals}

Édition électronique

URL : http://journals.openedition.org/edc/7178

DOI : 10.4000/edc.7178

ISSN : 2101-0366

Éditeur

Université de Lille

Édition imprimée

Date de publication : 1 décembre 2017

Pagination : 89-106

ISBN : 978-2-917562-18-5

ISSN : $1270-6841$

\section{Référence électronique}

Jean-Sébastien Vayre, "Une histoire de machines à recommander des biens de consommation : de I'Internet documentaire à l'Internet des données », Études de communication [En ligne], 49 | 2017, mis en ligne le 01 décembre 2019, consulté le 04 janvier 2020. URL : http://journals.openedition.org/edc/ 7178 ; DOI : 10.4000/edc.7178 
Une histoire de machines

à recommander des biens

de consommation : de l'Internet

documentaire à l'Internet des données

A History of Consumer

Recommendation Machines:

From the Internet of Documents

to the Internet of Data 
Depuis le succès d'Amazon et avec le développement du mouvement big data, les agents de recommandation connaissent une importante diffusion auprès des e-commerçants. Du point de vue de la sociologie économique, ces agents sont intéressants dans la mesure où ils constituent une forme relativement nouvelle de prescripteurs marchands. Aussi, afin de mieux saisir le rôle que ces intelligences artificielles jouent entre l'offre et la demande, nous proposons dans cet article de traiter le questionnement suivant : quelles sont les logiques de prescription des agents de recommandation numériques et comment ontelles évoluées dans le temps?

Mots-clés : agent de recommandation, prescription, marché, histoire des techniques, intelligence artificielle, big data, Internet.
With the success of Amazon and the development of the big data movement, automated recommendation agents have been increasingly adopted by online merchants. From a socio-economic perspective, these agents are interesting in that they constitute a relatively new form of commercial recommendation. In order to better understand the role that such artificial intelligence systems play, as mediators between supply and demand, we explore the prescriptive logic of digital recommendation agents as well as their evolution over time.

Keywords: recommendation agent, prescription, market, history of technology, artificial intelligence, big data, Internet. 


\section{Introduction}

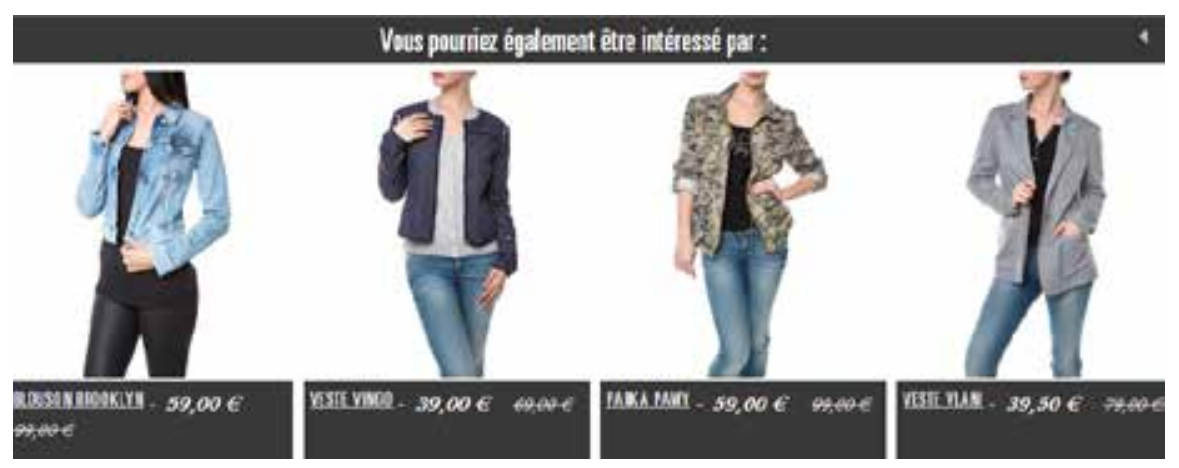

Figure 1 : exemple de prescriptions réalisées

par un agent de recommandation

Avec le mouvement big data que nous connaissons aujourd'hui, les technologies d'automatisation de la relation client connaissent un succès toujours plus important auprès des "professionnels du marché " (Cochoy et Dubuisson-Quellier, 2000) : elles se multiplient et gagnent en efficacité. De nombreuses entreprises proposent ainsi divers dispositifs d'intelligence artificielle qui ont pour fonction de permettre aux acteurs économiques d'automatiser une partie du travail qu'ils opèrent pour communiquer avec leurs clients. Parmi ces types d'instruments, les agents de recommandation de biens de consommation font partie des plus répandus. Ces agents sont des systèmes de traitement d'information qui ont pour fonction de prédire les préférences des consommateurs à partir des données que ceux-ci produisent durant leurs usages de l'Internet et de façon à leur proposer des produits susceptibles de les intéresser (cf. figure 1). Un peu à la manière du guide Michelin (Karpik, 2000), ou encore, de la presse consumériste (Mallard, 2000), ces agents peuvent être considérés comme des dispositifs de prescription : dans un contexte où l'information est surabondante et l'attention des consommateurs est limitée (Simon, 1971), ces technologies doivent permettre à ces derniers de surmonter plus aisément la crise cognitive que recouvre la construction de leur prise de décision d'achat (Keynes, 1973). Cependant, les agents de recommandation sont des prescripteurs d'un type particulier puisqu'à la différence de ceux étudiés par Armand Hatchuel (1995), ils sont des systèmes d'intelligence artificielle qui ne réalisent pas vraiment des prescriptions de fait, techniques ou de jugement, mais des prescriptions de choix'. Ils peuvent en ce sens être considérés comme des prescripteurs

1 Rappelons que chez Armand Hatchuel (1995), la prescription de fait consiste, pour le vendeur, à rappeler à l'acheteur des faits dont il a déjà connaissance. La prescription technique est différente dans la mesure où le vendeur apporte des connaissances nouvelles à l'acheteur. La prescription de jugement a lieu 
artificiels qui ont pour fonction d'architecturer les choix des consommateurs de façon à diminuer la charge attentionnelle qu'impliquent leurs activités de recherche d'information marchande.

Du point de vue de la sociologie économique, les agents de recommandation peuvent faire l'objet de deux interprétations. D'un côté, ils peuvent être considérés comme des manières de favoriser la participation des consommateurs aux services déployés par les entreprises pour communiquer sur leurs offres (cf. Maillet, 2007) : en produisant des recommandations aux consommateurs à partir de leurs données d'usages, ces agents leur permettent de contribuer à la fabrication des suggestions qui leur sont faites afin qu'elles soient perçues comme des informations utiles et non imposées. D'un autre côté, les agents de recommandation peuvent également être appréhendés comme des façons de mettre au travail les consommateurs en cherchant à exploiter économiquement les données que ces derniers produisent durant leurs activités de recherche d'information (cf. Dujarier, 2008) : en enrôlant les consommateurs dans le travail de prescription commerciale, ces technologies ont pour principale fonction de produire de la valeur marchande pour les e-commerçants. Dans cet article, nous prendrons une certaine distance vis-à-vis de ces deux types de discours. Autrement dit, avant de nous positionner trop rapidement en cherchant à expliquer pourquoi les agents de recommandation participent à aliéner ou à libérer les consommateurs, nous proposons de commencer par étudier comment ils font médiation entre l'offre et la demande. Pour ce faire, nous poserons le questionnement suivant : quelles sont les logiques de prescription des agents de recommandation numériques et comment ont-elles évolué dans le temps?

Afin de répondre à cette question, nous retracerons l'histoire de ces technologies d'un point de vue " internaliste " en montrant comment elles recouvrent des architectures cognitives particulières qui ont évolué au cours du temps. Pour réaliser cette tâche, nous nous appuierons sur un ensemble de documentations issues de la littérature grise ainsi que sur un travail de terrain que nous avons réalisé auprès de deux start-up commercialisant des agents de recommandation qui ont été implémentés au sein de plus d'une soixantaine d'e-commerçants chacun. Nous verrons alors comment les agents de recommandation à intelligence contextuelle (i.e., de troisième génération) performent des logiques de prescription qui sont plus flexibles que celles que mettent en action les agents à intelligences documentaire (i.e., de première génération) et sociale (i.e., de deuxième génération) : à la différence de ces deux derniers

quand l'acheteur a perdu tout repère : ce dernier s'en remet au jugement du vendeur. Ainsi, les agents de recommandation que nous étudions ici, en suggérant aux consommateurs des biens susceptibles de les intéresser, effectuent avant tout des prescriptions de choix. Notons que ces prescriptions peuvent alors prendre des formes qui rappellent les prescriptions de fait, techniques et de jugement en fonction des stratégies de navigation adoptées par les consommateurs et des niveaux de connaissances qu'ils ont du catalogue en ligne qu'ils sont en train de visiter. 
types d'agents, les règles d'inférence que produisent ceux dits à intelligence contextuelle ne sont pas déterminées, de façon statique, par leurs concepteurs, mais par eux-mêmes et de manière dynamique. Nous montrerons finalement comment cette troisième génération d'agent constitue une parfaite manifestation des solutions contemporaines développées pour lever le paradoxe qui consiste à articuler les logiques d'industrialisation et de personnalisation de la communication marchande à l'ère du numérique.

\section{1. \\ L'histoire des agents de recommandation}

Afin de pouvoir comprendre comment les agents de recommandation de troisième génération autorisent l'institution de logiques de prescription qui sont moins rigides que celles que performent les agents de première et de deuxième génération, nous souhaitons revenir sur leur histoire internaliste. Nous verrons ainsi que les trois types de prescripteurs artificiels que constituent ces technologies renvoient à des formes d'intelligence qui ont évolué avec le développement de l'Internet.

\subsection{Les agents de recommandation à intelligence documentaire}

Les agents de recommandation de première génération ont la particularité d'être fondés sur une analyse dite de contenu et renvoient au web 1.0 qui est souvent qualifié de passif (Ochi et al., 2010). Le web 1.0 repose sur le principe de la documentation qui, encore à la base de l'Internet d'aujourd'hui, peut être exposé comme suit : un concepteur produit un contenu qui est affiché sur Internet et qui est consulté par différents utilisateurs. Le principe de la documentation est ainsi sous-tendu par un modèle d'échange d'informations qui est de l'ordre de l'instruction puisque l'utilisateur ne participe, ni à la production du contenu du message, ni à la validation de sa valeur. Le web 1.0 est avant tout un outil de connaissance que l'utilisateur mobilise un peu à la manière d'une gigantesque bibliothèque : il s'en sert principalement pour s'informer (Fuchs et al., 2010). C'est un outil qui véhicule un ensemble de connaissances et de valeurs collectives qui s'imposent de l'extérieur aux individus et qui structurent leur vie en société.

Aussi, si les agents de recommandation de première génération sont souvent associés aux web 1.0, c'est parce qu'ils sont dotés d'une intelligence de type documentaire. Ces derniers sont en effet conçus à partir de la technique du filtrage fondé sur le contenu qui repose sur diverses formes d'association dites item-to-item (Chartron et Kembellec, 2014). Cela veut dire que les agents de première génération exploitent essentiellement les informations présentées dans les documents qui composent un site particulier. Par exemple, dans le cas du e-commerce, ces agents ont pour fonction de recommander aux utilisateurs des produits à partir des informations qui caractérisent ces mêmes biens. 
Plus précisément, ils ont pour objectif d'extraire les propriétés qualitatives et quantitatives qui sont propres à une marchandise afin de pouvoir proposer aux visiteurs du site d'autres produits bénéficiant de caractéristiques similaires.

\subsection{Les agents de recommandation à intelligence sociale}

La deuxième génération d'agents de recommandation est quant à elle fondée sur une analyse dite sociale qui renvoie à un web 2.0 souvent qualifié de collaboratif (Ochi et al., 2010). Comme son nom l'indique, le web 2.0 est une version augmentée du web 1.0. II a donc une fonction documentaire qui est centrale, mais qui est améliorée : le « 2.0 » permet d'indiquer qu'avec ce nouveau web, les utilisateurs ne sont plus contraints d'être des récepteurs passifs. Ils peuvent activement contribuer à la production des contenus et à la validation de leur valeur puisque cette nouvelle version de l'Internet ne repose plus seulement sur le principe de la documentation, mais aussi sur celui de la communication. À travers le développement des blogs, des forums et des réseaux sociaux, Internet n'est plus un simple outil de connaissance : c'est également un moyen d'expression. Le web 2.0 est de ce fait sous-tendu par un modèle d'échange d'informations qui est de l'ordre du partage et moins de celui de l'instruction. La gigantesque bibliothèque dont nous parlions en amont devient une sorte d'espace public que les utilisateurs mobilisent pour s'informer, mais aussi pour s'exprimer, se mettre en contact, se rencontrer et s'organiser (Fuchs et al., 2010). Ici, le web n'est plus, à tout le moins pas seulement, un stock de connaissances qui s'imposent de l'extérieur aux individus. Il est un outil d'expression et de mise en relation; de telle sorte qu'il permet à ses utilisateurs de participer à sa production. À l'ère du 2.0, Internet n'est pas qu'un ensemble de documents que les individus consultent afin de conduire leurs activités sociales; c'est aussi un instrument leur permettant de produire et de coordonner ces mêmes activités.

Un peu à la manière dont les agents de recommandation de première génération sont dotés d'une intelligence documentaire qui entre en résonnance avec les principes fondamentaux du web 1.0, les agents de deuxième génération ont une intelligence sociale qui fait écho aux principes fondamentaux du web 2.0. Car ces agents sont développés à partir de la technique du filtrage collaboratif qui repose sur le principe de la corrélation user-to-user (Chartron et Kembellec, 2014). Et ce principe consiste à recommander des produits à un utilisateur en fonction des évaluations effectuées par d'autres ayant des profils semblables. Ces évaluations peuvent être déclarées de manière implicite (e.g., fréquence, durée de consultation et achat d'un produit) ou explicite (e.g., attribution d'une note ou d'un like à un produit). Elles permettent alors de définir des scores ou, si l'on préfère, des votes qui peuvent être déterminés de nombreuses manières (cf. Chan, 1999) et qui sont considérés comme des indicateurs des appétences des utilisateurs. Notons toutefois que la technique du filtrage collaboratif n'est pas nécessairement réalisée à partir des seules préférences plus ou moins explicitement déclarées par les utilisateurs. Des 
données sociodémographiques peuvent, par exemple, venir compléter les indicateurs d'appétences des utilisateurs. Sur le plan théorique, ces données sont potentiellement capables d'affiner le calcul des distances existantes entre les différents utilisateurs représentés dans la base de données.

\subsection{Les agents de recommandation à intelligence contextuelle}

La troisième génération d'agents de recommandation est fondée sur une analyse hybride qui mélange les techniques de filtrage basé sur le contenu, de filtrage collaboratif et/ou les technologies dites de machine learning (i.e., $\mathrm{d}^{\prime}$ apprentissage artificiel $^{2}$ ) qui ont plus récemment été élaborées avec le développement des données issues des capteurs de l'Internet des objets (cf. Vayre, 2016). Cette troisième génération renvoie au web 3.0 qui est souvent qualifié de web des données, ou encore, de web sémantique (Ochi et al., 2010). Il est important de souligner que cette version 3.0 de l'Internet est récente puisqu'elle est, encore aujourd'hui, en train d'être développée. Comme dans le cas du web 2.0, le web 3.0 repose sur les principes de la documentation et de la collaboration. Toutefois, avec le développement du big data, il recouvre également une troisième dimension qui est celle de la " servuction ${ }^{3}$ (Eiglier et Langeard, 1987) dans le sens où, à travers les usages quotidiens que l'utilisateur fait des sites disponibles sur Internet et des objets connectés, celui-ci tend à participer, de manière toujours plus intense, non consciente et involontaire, à la production des différents services disponibles sur la toile. La multiplication des agents de recommandation sur le web en constitue un bon exemple. Le web 3.0 peut de ce fait être considéré comme sous-tendu par un modèle d'échange d'informations qui est de l'ordre de la coproduction : avec l'intérêt croissant que les acteurs du web portent aux données, Internet tend à devenir une sorte de grand espace de services plus ou moins personnalisés que l'utilisateur produit dans le même temps qu'il les utilise (Cardon, 2015). À I'instar de leurs ancêtres de première et de deuxième générations, les agents de recommandation de troisième génération sont dotés d'une intelligence qui, dans une certaine mesure, est propre au web 3.0. Ces derniers sont souvent des formes hybrides des systèmes de filtrage fondé sur le contenu, de filtrage collaboratif, mais aussi de bien d'autres types d'algorithmes plus ou moins originaux (Chartron et Kembellec, 2014 ; Vayre, 2016). En effet, les concepteurs de ces agents disposent actuellement d'une importante diversité de technologies d'apprentissage artificiel leur permettant de donner du sens à des données qui, jusque-là, apparaissaient ne comporter aucune valeur. À condition de dispo-

2 Les professionnels parlent également d'apprentissage statistique voire, dans certains cas, d'apprentissage automatique.

3 La notion de servuction est un néologisme inventé par Pierre Eiglier et Éric Langeard (1987) qui rassemble les notions de service et de production afin de souligner comment les utilisateurs d'un service peuvent contribuer à sa production. 
ser des moyens techniques nécessaires, ces technologies peuvent permettre aux agents de recommandation de traiter, en temps quasi-réel, un nombre très important de données qui semblaient, jusqu'ici, inexploitables et d'une façon qui est parfois assez originale. Compte tenu des masses de données qu'il est actuellement possible de recueillir sur les pratiques des utilisateurs et du développement des technologies permettant de les traiter, les agents de recommandation de troisième génération détiennent, pour la plupart d'entre eux, une intelligence que l'on peut qualifier de documentaire, de sociale, mais aussi, de contextuelle. Pourquoi ajouter le qualificatif « contextuel »? Parce que, dans le sens des travaux de Dominique Cardon (2015), grâce aux big data, les agents de troisième génération sont capables de définir plus précisément que leurs prédécesseurs les comportements des utilisateurs ainsi que les cadres socio-matériels à l'intérieur desquels ces derniers sont déployés ${ }^{4}$.

\section{2.}

\section{Deux agents de recommandation à intelligence contextuelle}

Ces dernières années, avec le développement du mouvement big data, de nombreuses start-up se sont lancées dans la conception d'agents de recommandation à intelligence contextuelle. Dans le cadre d'une recherche qui s'est déroulée de 2012 à 2015, nous avons rencontré les principaux acteurs toulousains engagés dans la conception de ces machines. Nos investigations nous ont alors permis de mieux comprendre comment ces agents de recommandation de troisième génération peuvent recouvrir de nouvelles logiques de prescription. Dans cette section nous proposons d'illustrer cette diversité à partir de la présentation des agents de recommandation développés par les sociétés DataCrawler et DataCognitive ${ }^{5}$. Nous chercherons de cette manière à rendre compte de la façon dont les intelligences de ces deux agents autorisent l'institution de logiques de prescription qui sont plus flexibles que celles véhiculées par les agents à intelligences documentaire et sociale. Ce faisant, nous montrerons comment, du point de vue de leurs architectures cognitives, l'agent de DataCrawler et celui de DataCognitive recouvrent des logiques de prescription qui consistent principalement à maximiser l'utilité économique des activités de recherche d'information des consommateurs dans le même temps qu'elles doivent autoriser l'exploitation économique, par les e-commerçants, des traces d'usages produites par leurs clients.

4 Rappelons que pour Dominique Cardon (2015), les algorithmes d'apprentissage artificiel peuvent être compris comme des machines à « ingurgiter des contextes".

5 DacaCrawler et DataCognitive sont des noms fictifs que nous avons inventés afin de protéger l'anonymat des acteurs considérés. 


\subsection{L'agent de recommandation de DataCrawler}

DataCrawler est une société de programmation informatique qui a été créée en septembre 2012. C'est une petite start-up toulousaine qui est composée, en 2013, de six associés. L'entreprise est, à ce moment-là, spécialisée dans la conception d'outils informatiques à destination des acteurs du e-commerce. Plus précisément, elle propose trois technologies : un moteur de recherche pour le e-commerce, un agent de recommandation de biens de consommation et un système de personnalisation de courriel à destination des consommateurs. Au début de l'année 2014, DataCrawler compte plus de soixante-dix clients. À cette même période, le directeur de DataCrawler décide de "pivoter " en s'engageant dans un autre projet de création d'entreprise avec un des co-fondateurs d'une des plus importantes sociétés françaises de marketing automatique. II se sépare progressivement de ses six associés et laisse fonctionner la société, par elle-même, pendant un peu plus de deux ans. En juillet 2016, la société DataCrawler est fermée. La technologie proposée par cette société qui a été la plus vendue est son agent de recommandation. Nous allons voir que ce dernier est un agent de troisième génération relativement classique dans la mesure où il repose sur l'articulation de cinq algorithmes de recommandation dont l'hybridation est déterminée par un algorithme d'apprentissage.

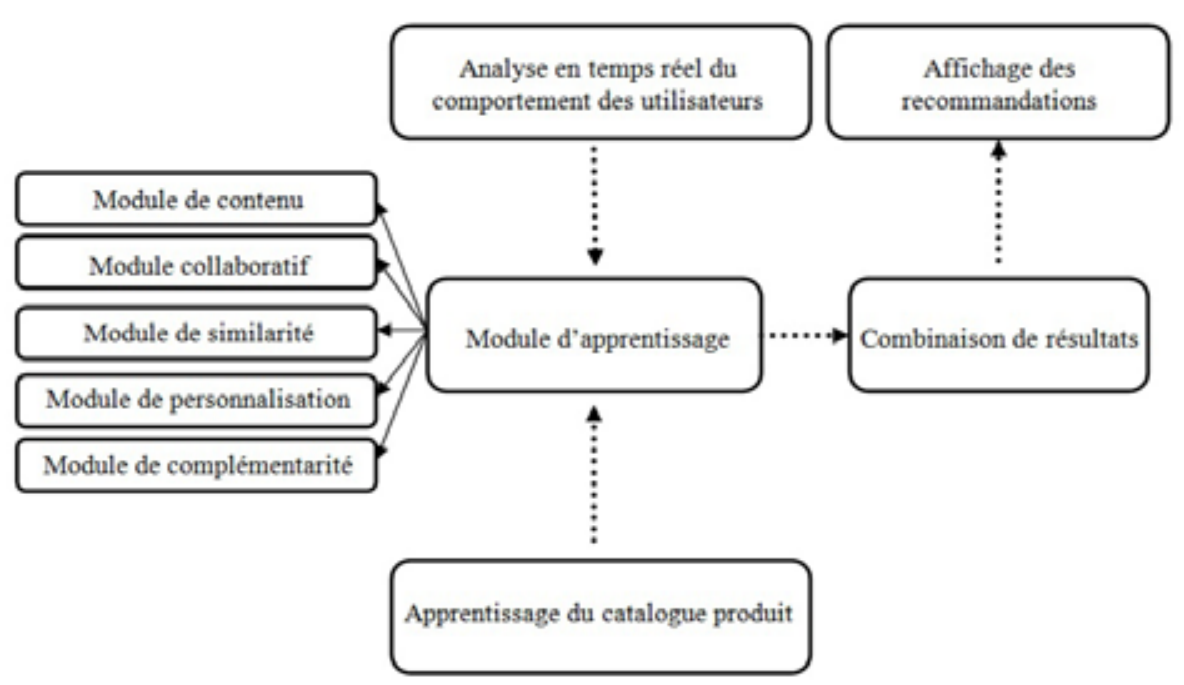

Figure 2 : architecture cognitive de l'agent de recommandation de DataCrawler 
Le premier algorithme de recommandation est classique. C'est un système de filtrage basé sur le contenu. II repose donc sur le principe de la corrélation item-to-item dont nous avons parlé plus haut. Le deuxième algorithme de recommandation est également assez conventionnel puisqu'il s'agit d'un algorithme de filtrage collaboratif qui est basé sur le principe de la corrélation user-to-user que nous avons aussi présenté. Le troisième algorithme de recommandation est un algorithme original qui a été conçu par l'entreprise DataCrawler. Son dirigeant le qualifie de similarité basée sur la rareté. Cet algorithme dit sémantique consiste à analyser les contenus des descriptions et les images des produits vendus sur le site d'e-commerce considéré. La singularité de cet algorithme est alors de repérer les unités informationnelles qui sont à la fois les plus pertinentes et les plus spécifiques à un même produit (e.g., le terme "rétropédalage ", ou encore, une selle en cuir marron foncé avec des rivets chromés sur un site de vente de vélo de route français). Le quatrième algorithme de recommandation est également une conception propre à l'entreprise DataCrawler. II est dit de personnalisation. Cet algorithme ressemble à celui de similarité basée sur la rareté puisqu'il fonctionne également à partir d'une analyse des contenus des descriptions et des images des produits. Cependant, ici, la singularité du produit à moins d'importance que la récurrence des actions du consommateur. Par exemple, dans le cas d'un site de vente de vélo de route, si un consommateur clique plusieurs fois sur des vélos homme de couleur noir, l'algorithme de personnalisation va prédire que ce consommateur s'intéresse aux vélos homme de couleur noir, même si ces deux caractéristiques ne sont pas rares comparativement aux autres produits disponibles. Le cinquième algorithme de recommandation est un dérivé de l'ensemble des quatre autres algorithmes. II est dit de complémentarité. Cet algorithme hybride les algorithmes de filtrage collaboratif, de similarité basée sur la rareté et de personnalisation. Il consiste à retenir, en fonction de la navigation du consommateur, les produits bénéficiant de la plus forte prédiction du point de vue de ces trois algorithmes et qui sont les moins similaires aux produits de référence (i.e., celui correspondant à la fiche produit consultée par le consommateur ; cf. l'algorithme de filtrage basé sur le contenu). L'algorithme d'apprentissage prend en charge l'hybridation des cinq algorithmes présentés précédemment. En d'autres termes, c'est lui qui détermine une combinaison gagnante plus ou moins durable et généralisable des cinq algorithmes de recommandation exposés en amont en fonction des trois critères de performance que sont : le taux de conversion (i.e., de transformation de visiteurs en acheteurs), le nombre de clics réalisés par les consommateurs sur les recommandations et la durée de consultation de ces recommandations.

\subsection{L'agent de recommandation de DataCognitive}

À l'instar de la société DataCrawler, l'entreprise DataCognitive est une start-up toulousaine spécialisée dans le champ de l'informatique commerciale. DataCognitive a été créée en 2010 à partir de la rencontre entre deux 
spécialistes de l'édition de logiciels pour le e-business et un informaticien qui travaillait sur le développement d'un processeur de traitement de situation ${ }^{6}$. De cette rencontre est né, en 2011, un premier prototype d'application de ce processeur au domaine du e-commerce. La première version de cette technologie est commercialisée en 2013 et est progressivement adoptée par plusieurs e-commerçants français. Elle se décline en quatre applications différentes : une première qui vise à personnaliser les campagnes de communication des e-commerçants; une seconde qui est spécialisée dans la recommandation de produits de consommation ; une troisième qui doit permettre de réduire les abandons de panier ; et, une quatrième qui consiste à personnaliser les courriels envoyés aux clients. À la différence de la société DataCrawler, DataCognitive continue, encore aujourd'hui, de se développer. En 2017, elle est implantée à Londres, à Paris et à Toulouse où se fait l'essentiel de la recherche et développement.

Le fonctionnement du processeur de traitement de situation est aussi complexe qu'original dans le champ de la recommandation automatique. II constitue ce que l'on appelle en intelligence artificielle une architecture cognitive hybride, c'est-à-dire un système de traitement d'information qui associe une architecture cognitive de type ascendante (i.e., bottom-up) et une architecture cognitive de type descendante (i.e., top-down ; cf. Smolensky et al., 1992). Comme le souligne son concepteur dans la demande de brevet qu'il a soumise à I'Institut National de la Propriété Intellectuel (INPI) en 2010, le processeur de traitement de situation n'est donc « ni connexionniste [soit, une architecture cognitive ascendante] ni computationniste [soit, une architecture cognitive descendante] ". Il est les deux à la fois : " le dispositif selon l'invention est asynchrone, non déterministe et s'avère ni connexionniste ni computationniste puisque sa puissance est à la fois dans sa structure et dans sa logique. II accepte un fonctionnement séquentiel comme parallèle " (Demande de brevet du processeur de traitement de situation, 2010). Plus précisément, le processeur de traitement de situation est composé : d'une part, d'un intégrateur, d'une mémoire situationnelle et d'une mémoire de masse qui forment un système de traitement ascendant des données (i.e., de type connexionniste) ; et, d'autre part, d'un concentrateur, d'un moteur d'inférence et d'une bibliothèque qui composent un système de traitement descendant des données (i.e., de type computationniste).

6 L'appellation « processeur de traitement de situation » est fictive. Nous l'avons inventée afin de ne pas faire de publicité positive ou négative à l'innovation en question ainsi qu'à son concepteur. 


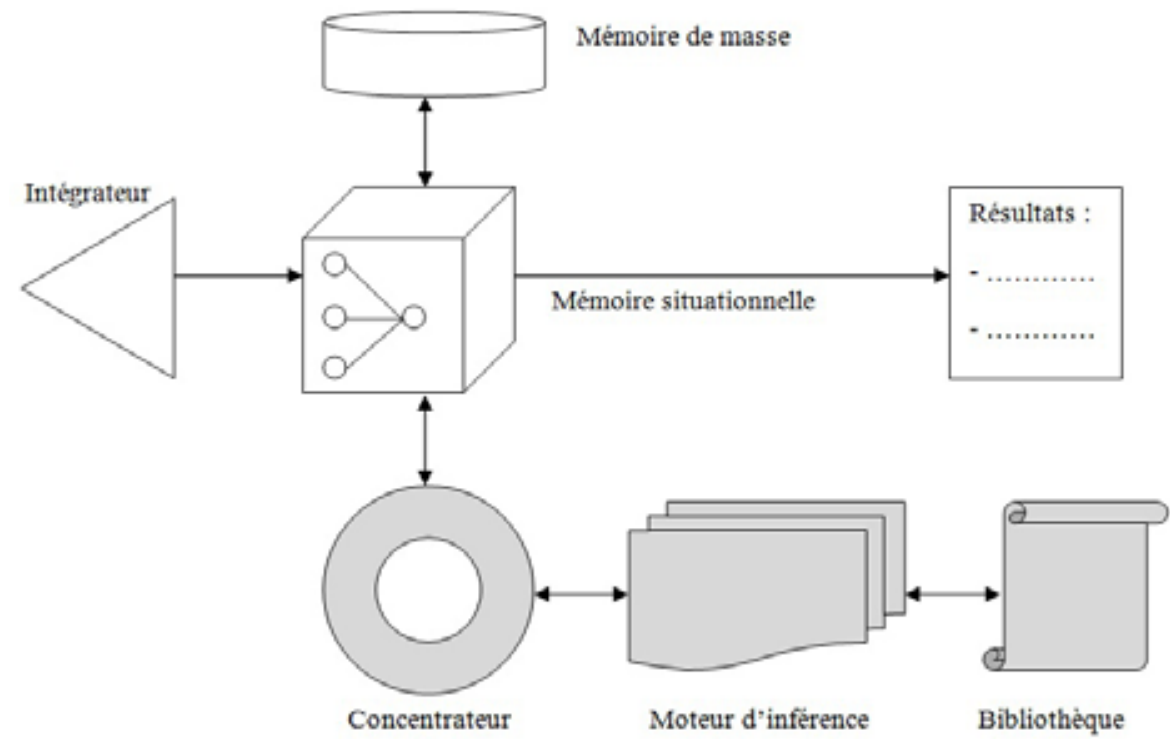

Figure 3 : architecture cognitive de l'agent de recommandation de DataCognitive

Faire une description détaillée du fonctionnement du processeur de traitement de situation demanderait un article à lui seul. Nous proposons donc d'en présenter les seuls principes de base. Dans les cas de Data Cognitive, le processeur de traitement de situation est mobilisé afin de manipuler les données de navigation des utilisateurs d'un site d'e-commerce ainsi que les documents qui le composent dans le but de pouvoir personnaliser l'expérience des consommateurs en leur proposant différentes recommandations de produits et/ou de services. Aussi, l'intégrateur est un module qui permet de récupérer l'ensemble des données qui vont informer l'agent de recommandation sur la situation de navigation d'un visiteur (e.g., adresse IP, profil de navigation, clics, like, type de page consultée, stratégie de communication exposée, etc.). Une fois ces données récupérées, l'intégrateur les répartit au sein de la mémoire situationnelle. Cette mémoire fonctionne de manière relativement analogue aux technologies de deep learning (i.e., aux réseaux de neurones profonds) : en fonction du flux de données qu'elle reçoit de l'intégrateur, la mémoire situationnelle du processeur lui permet de réaliser différents schémas de connexion. Et, à partir de ces schémas, l'agent de recommandation peut apprendre à induire des généralisations qui peuvent être représentées par des situations du type :

Les nouveaux visiteurs qui consultent des pages produits présentant des disques durs ont tendance à cliquer sur des recommandations de housses de disques durs.

Ces généralisations sont stockées dans la mémoire de masse. Par le biais du concentrateur et du moteur d'inférence, elles sont traduites sous la forme 
de symboles et de règles qui prennent place dans la bibliothèque. Le concentrateur est un module de gestion des ressources calculatoires du processeur de traitement de situation. Il lui permet de prioriser ses activités inférentielles. La bibliothèque est la principale interface qui permet à l'homme de communiquer avec l'agent : grâce à elle, l'e-commerçant peut contrôler les apprentissages de la machine. Il peut également décider d'inverser le fonctionnement du processeur de traitement situation en introduisant en son sein différentes connaissances plus ou moins expertes de façon à cadrer ses activités inférentielles. Ce faisant, l'e-commerçant fait entrer l'agent dans des logiques de prescription qui ne sont plus seulement inductives (i.e., bottom-up), mais aussi déductives (i.e., top-down). Au final, l'ensemble du fonctionnement de l'agent de DataCognitive est supervisé de façon à optimiser les mêmes critères de performance que dans le cas de l'agent de DataCrawler, c'est-à-dire le taux de conversion des visiteurs en acheteurs, le nombre de clic recommandation et la durée de consultation de ces recommandations.

\subsection{De nouvelles logiques de prescription}

Du point de vue de la sociologie économique, les deux descriptions que nous venons de proposer des agents de DataCrawler et de DataCognitive sont intéressantes dans la mesure où elles mettent en avant que ces technologies recouvrent des logiques de prescription qui sont différentes de celles qui sont traditionnellement associées aux agents de recommandation à intelligences documentaire et sociale. Car, un peu à la manière des algorithmes appliqués à la finance qu'étudie Fabian Muniesa (2003), les agents de recommandation de première et de deuxième générations performent des points de vue particuliers sur la façon dont il est possible de comprendre l'organisation des marchés. Les agents à intelligence documentaire mettent en action la logique selon laquelle, pour prescrire un bien à un consommateur, il est important de connaître les différentes propriétés des produits qu'il a déjà plus ou moins implicitement déclarés comme intéressants afin de lui en proposer d'autres qui sont similaires. Les agents à intelligence sociale font quant à eux fonctionner la logique selon laquelle, pour prescrire un bien à consommateur, l'important est de connaître les préférences d'autres consommateurs ayant des profils semblables. Notons qu'à la différence de l'agent de DataCognitive, l'agent de DataCrawler performe également ce type de logique puisque, comme nous l'avons vu, son architecture repose sur un système d'hybridation des deux logiques de prescription que nous venons de mentionner, mais aussi de trois autres qui renvoient à des principes plus originaux (cf. section II.1). II n'en reste pas moins qu'à l'instar de l'agent de DataCognitive, cet agent recouvre une logique générale de prescription beaucoup plus flexible que celles qui sont associées à ceux de première et de deuxième générations : son système d'apprentissage lui permet de déterminer, par lui-même et de façon dynamique, la règle d'hybridation des divers algorithmes qui composent son architecture cognitive. Soulignons que, toujours d'un point de vue général, la logique de prescription que performe l'agent de 
DataCognitive est encore moins rigide que celle que met en action l'agent de DataCrawler puisqu'elle ne repose même plus sur l'hybridation d'algorithmes qui renvoient à des hypothèses fortes sur la façon dont il est possible de déterminer les préférences des consommateurs. Son architecture cognitive lui permet de dégager la totalité des règles d'associations qui lui permettent de prédire ces appétences. Dans le sens des réflexions de Milad Doueihi (2017), il est ainsi important de comprendre que les agents de DataCrawler et de DataCognitive n'expriment plus, à tout le moins plus seulement, les logiques de prescription que leur prêtent ceux qui les conçoivent : ils performent des représentations sur la manière dont il est possible d'apprendre à découvrir et/ ou à articuler automatiquement ces logiques.

Cela veut dire qu'avec le développement des technologies d'apprentissage artificiel, les agents de recommandation de troisième génération sont dotés d'un pouvoir de prescription qui, a minima en partie, leur est propre. Tout l'avantage de leur intelligence contextuelle est en effet de leur permettre de déterminer seuls les règles d'inférence qui autorisent leurs activités de prescription de choix. Ces agents contribuent en ce sens à l'institution d'un nouveau mode de participation des consommateurs aux services qu'ils leur offrent dans le sens où leur intelligence les rend plus attentifs à la singularité des clients. Dans une certaine mesure, les agents de DataCrawler et de DataCognitive ont par là même pour fonction de libérer les consommateurs, au moins partiellement, des effets performatifs qui sont associés à la mise en prescription des représentations que les acteurs de l'offre peuvent se faire de la demande. Pour autant, ce gain de liberté n'est pas synonyme d'émancipation. Car l'autonomie des agents de recommandation à intelligence contextuelle est limitée. Bien que les agents de DataCrawler et de DataCognitive recouvrent des méthodes d'apprentissage artificiel qui ne sont pas toutes supervisées, nous avons vu que les architectures cognitives qu'ils composent sont élaborées de façon à ce que leurs activités de prescription optimisent les ventes de ceux qui les implémentent. Pour le dire autrement, toute l'autonomie de calcul dont bénéficient ces agents est orientée de façon à favoriser les intérêts économiques des e-commerçants qui les intègrent à leur catalogue en ligne. Or, cette orientation pourrait être autre : il serait par exemple tout à fait possible d'imaginer que le sens du travail de prescription de ces agents soit orienté de façon, non plus à maximiser l'achat de biens par les consommateurs, mais à minimiser leur désorientation. Les logiques de prescription apprises par les agents de DataCrawler et de DataCognitive prendraient alors des allures bien différentes. Mais, pour le moment, si la flexibilité des logiques de prescription de ces deux agents de recommandation a pour fonction de favoriser la liberté de choix des consommateurs, c'est aussi dans l'objectif d'améliorer les performances économiques des e-commerçants qui les implémentent. 


\section{Conclusion}

De façon traditionnelle, les agents de recommandation sont des technologies qui ont eu pour fonction de lever le paradoxe qu'implique la gestion d'un mode de communication à la fois industriel et personnalisé. Ils font partie de ces instruments de gouvernance libérale qui n'ont plus pour objectif de discipliner les individus par la contrainte, mais plutôt d'orienter leur comportement en cherchant à maximiser l'utilité des environnements qu'ils traversent (Cardon, 2017) : avec les agents de recommandation, il s'agit moins de jouer sur les joueurs que sur les règles du jeu. L'histoire internaliste que nous avons proposé nous apprend alors que, malgré leur connivence étroite avec l'idéologie du laisser-faire, ces technologies n'en restent pas moins des outils de cadrage qui véhiculent des logiques particulières de prescription. Ces logiques renvoient globalement à deux types de représentation : d'une part, celle qui porte sur la façon dont il est possible de définir et prédire les préférences des utilisateurs ; et, d'autre part, celle qui porte sur la manière dont il est possible d'instrumenter ces préférences de façon à servir les intérêts des demandeurs et des offreurs.

Nous avons alors mis en avant comment le double rôle qu'ont pris en charge les agents de recommandation a évolué avec le développement de l'Internet. Les logiques de prescription des agents de première et de deuxième générations renvoient à des formes d'intelligences qui sont propres à l'Internet 1.0 et 2.0 : elles sont, respectivement, de type documentaire et de type social. La section I nous a ainsi permis de montrer que les logiques de prescription des agents de première et de deuxième générations recouvrent des définitions des préférences des utilisateurs qui sont assez rigides ; de telle sorte qu'au travers des recommandations réalisées par ces agents, il s'agit moins de s'adapter au monde que de le performer. Aussi, les agents de recommandation de troisième génération sont bien plus flexibles, sur le plan cognitif, que ceux de première et de deuxième génération. L'agent de DataCrawler et celui de DataCognitive en constituent deux bons exemples dans la mesure où, comme nous l'avons souligné dans la section II, ils sont dotés d'une capacité d'apprentissage qui leur permet d'adapter, de façon plus ou moins poussée, leurs recommandations en fonction des représentations qu'ils se font des consommateurs et de leurs environnements d'action. Cependant, nous avons également mis en avant que cette intelligence, que nous avons qualifiée de contextuelle, est, à tout le moins en bout de chaîne, toujours supervisée par les e-commerçants. À partir du cas de l'agent de DataCrawler et de celui de DataCognitive, la section II nous a alors permis de pointer que cette supervision est instituée par le biais du choix des critères d'optimisation qui permettent l'auto-évaluation dynamique, par la machine, de la qualité de ses apprentissages.

En somme, les agents de recommandation que nous connaissons actuellement tendent à être dotés d'une intelligence nouvelle qui peut prendre des formes relativement diverses et qui leur permet d'associer, de façon toujours plus intime, la mise au travail et la participation des consommateurs (Maillet, 2007 ; 
Dujarier, 2008). Car, si ces prescripteurs artificiels sont aujourd'hui capables d'apprendre par eux-mêmes à personnaliser les environnements numériques marchands des consommateurs en fonction des données que ces derniers produisent durant leurs activités de recherche d'information commerciale, l'ensemble de leur travail inférentiel est quasi-systématiquement orienté en fonction des intérêts financiers des entreprises qui les implémentent. Et c'est précisément en ce sens qu'avec les agents de recommandation à intelligence contextuelle, la participation et la mise au travail des consommateurs s'entremêlent pour donner forme à de mêmes logiques de prescription. 
Bibliographie

Cardon D. (2015). À quoi rêvent les algorithmes. Nos vies à I'heure des big data, Paris, SeuilLa République des idées.

Cardon D. (2017). Le bazar et les algorithmes. La société des calculs numériques, Paris, Université Paris-Est.

Chan P. (1999). "A non-invasive learning approach to building web user profiles". In Kdd-99 Workshop on Web Usage Analysis and User Profiling. Disponible sur http://cs.fit. edu/ pkc/papers/webkdd99.pdf (page consultée le 20 février 2017).

Chartron G., Kembellec G. (2014). "Introduction générale aux systèmes de recommandation ". In Kembellec G., Chartron G., Saleh I. (dir.), Les moteurs et systèmes de recommandation, Londres, ISTE Édition, p. 23-42.

Cochoy F., Dubuisson-Quellier S. (2000). «Introduction. Les professionnels du marché : vers une sociologie du travail marchand ». In Sociologie du travail, vol. $42, n^{\circ} 3$, p. 359-368.

Doueihi M. (2017). « Environnement et milieu. Du vivant au numérique ". In Collège des Bernardins, L'humain face au défi du numérique, Séminaire de recherche du 7 mars, Paris. Disponible sur https:// www.collegedesbernardins.fr/ recherche/comptes-rendus-chaire-
Ihumain-au-defi-du-numerique (page consultée le 14 juin 2017).

Dujarier M.-A. (2008). Le travail du consommateur. De McDo à eBay, comment nous coproduisons ce que nous achetons, Paris, La Découverte.

Eiglier P., Langeard E. (1987). Servuction : le marketing des services, Cachan, Ediscience International.

Fuchs C., Hofkirchner W., Schafranek M., Raffl C., Sandoval M. (2010). "Theoretical foundations of the web: cognition, communication, and co-operation. Towards an understanding of web 1.0, 2.0, 3.0". In Future Internet, vol. 2, n 1, p. 41-59.

Hatchuel A. (1995). « Les marchés à prescripteurs ». In Verin H., Jacob A. (dir.), L'inscription sociale du marché. Paris, Harmattan, p. 205-225.

Karpik L. (2000). " Le guide rouge Michelin ». In Sociologie du travail, vol. $42, n^{\circ} 3$, p. 369-389.

Keynes J. (1973). The Collected Writting, VIII, A Treatise on Probability, Londres, Macmillan.

Maillet T. (2007). Génération participation : de la société de consommation à la société de participation, Paris, M21 Edition.

Mallard A. (2000). «La presse de consommation et le marché. Enquête sur le tiers consumériste ». In Sociologie du travail, vol. 42, n³, p. 391-409. 
Ochi P., Rao S., Takayama L., Nass

C. (2010). "Predictors of User

Perceptions of Web Recommender

Systems: How the Basis for Gen-

erating Experience and Search

Product Recommendations Affects

User Responses". In International

Journal of Human-Computer

Studies, vol. 68, p. 472-482.

Simon H.A. (1971). “Designing

Organizations for an Informa-

tion-Rich World". In Greenberger M.

(dir.), Computers, Communications

and the Public Interest, Baltimore,

John Hopkins Press, p. 37-72.

Smolensky P., Legendre G., Miyata

Y. (1992). Principles for an Integrated

Connectionist/Symbolic Theory

of Higher Cognition. University

of Colorado, Computer Science

Technical Report. Disponible sur

http://scholar.colorado.edu/cgi/

viewcontent.cgi?article $=1574 \&$ -

context=csci_techreports (page

consultée le 20 février 2017).

Vayre J.-S. (2016). Des machines à produire des futurs économiques : sociologie des intelligences artificielles marchandes à l'ère du big data. Thèse de doctorat, Université Toulouse Jean Jaurès. Disponible sur https://www.researchgate.net/ publication/311128070_Des_machines_a_produire_des_futurs_economiques_sociologie_ des_intelligences_artificielles_marchandes_a_l'ere_du_big_data (page consultée le 20 février 2017). 